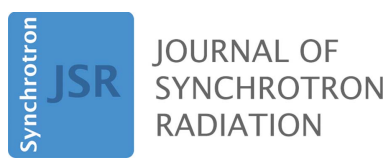

ISSN 1600-5775

Received 16 October 2018

Accepted 7 March 2019

Edited by I. Schlichting, Max Planck Institute for Medical Research, Germany

${ }^{1}$ This article will form part of a virtual special issue on X-ray free-electron lasers.

Keywords: FEL; X-ray; pump-probe; time-resolved.

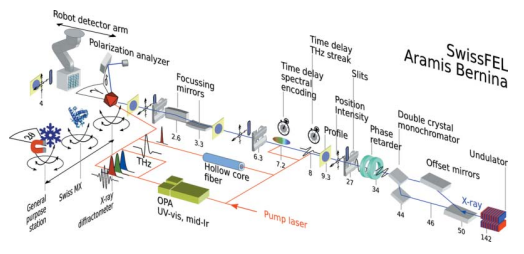

OPEN $\odot$ ACCESS

\section{Experimental station Bernina at SwissFEL: condensed matter physics on femtosecond time scales investigated by $X$-ray diffraction and spectroscopic methods ${ }^{1}$}

\author{
Gerhard Ingold, ${ }^{\mathrm{a} *}$ Rafael Abela, ${ }^{\mathrm{a}}$ Christopher Arrell, ${ }^{\mathrm{a}}$ Paul Beaud, ${ }^{\mathrm{a}}$ Pirmin Böhler, \\ Marco Cammarata, ${ }^{\mathrm{b}}$ Yunpei Deng, ${ }^{\mathrm{a}}$ Christian Erny, ${ }^{\mathrm{a}}$ Vincent Esposito, ${ }^{\mathrm{a}}$ \\ Uwe Flechsig, ${ }^{a}$ Rolf Follath, ${ }^{\text {a }}$ Christoph Hauri, ${ }^{a}$ Steven Johnson, ${ }^{\mathrm{c}}$ Pavle Juranic, ${ }^{\mathrm{a}}$ \\ Giulia Fulvia Mancini, ${ }^{\mathrm{d}}$ Roman Mankowsky, ${ }^{\mathrm{a}}$ Aldo Mozzanica, ${ }^{a}$ \\ Roland Alex Oggenfuss, ${ }^{a}$ Bruce D. Patterson, ${ }^{a}$ Luc Patthey, ${ }^{a}$ Bill Pedrini, ${ }^{a}$ \\ Jochen Rittmann, ${ }^{\mathrm{a}}$ Leonardo Sala, ${ }^{\mathrm{a}}$ Matteo Savoini, ${ }^{\mathrm{c}}$ Cristian Svetina, ${ }^{\mathrm{a}}$ \\ Thierry Zamofing, ${ }^{a}$ Serhane Zerdane ${ }^{a}$ and Henrik Till Lemke ${ }^{a *}$
}

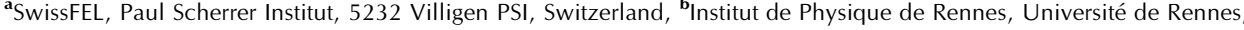
35042 Rennes CEDEX, France, ' Institute for Quantum Electronics, Eidgenössische Technische Hochschule (ETH) Zürich, CH-8093 Zurich, Switzerland, and ${ }^{\mathbf{d}}$ Ecole Polytechnique Fédérale de Lausanne, CH-1015 Lausanne, Switzerland. *Correspondence e-mail: gerhard.ingold@psi.ch, henrik.lemke@psi.ch
}

The Bernina instrument at the SwissFEL Aramis hard X-ray free-electron laser is designed for studying ultrafast phenomena in condensed matter and material science. Ultrashort pulses from an optical laser system covering a large wavelength range can be used to generate specific non-equilibrium states, whose subsequent temporal evolution can be probed by selective X-ray scattering techniques in the range $2-12 \mathrm{keV}$. For that purpose, the X-ray beamline is equipped with optical elements which tailor the X-ray beam size and energy, as well as with pulse-to-pulse diagnostics that monitor the X-ray pulse intensity, position, as well as its spectral and temporal properties. The experiments can be performed using multiple interchangeable endstations differing in specialization, diffractometer and X-ray analyser configuration and load capacity for specialized sample environment. After testing the instrument in a series of pilot experiments in 2018, regular user operation begins in 2019.

\section{Introduction}

Hard X-ray free-electron lasers (XFELs) (Emma et al., 2010; Ishikawa et al., 2012; Kang et al., 2017) provide ultrashort $\mathrm{X}$-ray pulses which combine the sensitivity to molecular and crystal structures, as well as to core electron levels, with sufficient time resolution to resolve electronic transitions and atomic motions. Over the last decades, high-flux and highrepetition-rate synchrotron X-ray sources have revolutionized the insights into static electronic and nuclear structures of condensed matter materials, by combining elastic and inelastic diffraction methods with spectroscopic sensitivity to specific electronic and magnetic states of selected elements in a material (Kim et al., 2011; Gibbs et al., 1988; Murakami et al., $1998 a, b)$. Such methods have revealed material structures in bulk and surface, of nanometre-sized objects and crystals, as well as the complicated interplay of electronic, magnetic and nuclear structure in strongly correlated electron systems. The femtosecond $\left(10^{-15} \mathrm{~s}\right)$ time resolution provided at XFELs opens experimental access to short-lived out-of-equilibrium states which determine transitions between charge states, 
Table 1

X-ray FEL parameters at the SwissFEL Bernina instrument.

\begin{tabular}{ll}
\hline Undulator beamline & Aramis \\
Electron energy & Up to $5.8 \mathrm{GeV}$ \\
Electron bunch charge & $20-200 \mathrm{pC}$ \\
Photon energy & $1.77-12.4 \mathrm{keV}$ \\
Pulse energy & $1 \mathrm{~mJ}$ \\
Pulse duration & $0.1-100 \mathrm{fs}$ \\
Repetition rate & $100 \mathrm{~Hz}$ \\
\hline
\end{tabular}

magnetic and electronic phases and atomic/crystalline structures (Murakami et al., 1998b; Trigo et al., 2013; Beaud et al., 2014; Clark et al., 2013; Singer et al., 2016). The results indicate that additional insight into complex material functions can be gained in the time domain. Materials with complex interactions of charge-transfer energy, magnetic exchange, JahnTeller splitting, hopping integral or Hubbard interaction ( $0.15-4 \mathrm{eV})$ exhibit interesting phenomena like ferro- and antiferromagnetism, metal-to-insulator transitions, multiferroics, charge and spin density waves (CDW, SDW), colossal magnetoresistance (CMR), high- $T_{\mathrm{c}}$ superconductivity or topological insulation. When perturbing the equilibrium by external parameters while following the involved degrees of freedom in the time domain, their coupling and relevance for the phenomenon can be studied in much greater detail. FELs represent here an important complementary tool as they allow sensitive X-ray methods to be applied in the femtosecond time domain, like resonant and non-resonant X-ray diffraction (tr-RXRD) of elastic and inelastic scattering (tr-RIXS) which are selective to electronic, magnetic and nuclear structure.

The SwissFEL Aramis XFEL (Milne et al., 2017) provides $\mathrm{X}$-ray pulses in the energy range from 1.77 to $12.4 \mathrm{keV}$ (Table 1 ), which covers the $K$-edges of elements from $\mathrm{P}$ to As ( $Z=15$ to 33 ), the $L$-edges of most $4 d$ and $5 d$ materials ( $\mathrm{Rb}$ to $\mathrm{Au})$ as well as the $M$-edges from Dy $(Z=66)$ onward. The pulse length theoretical shorter limit down to $0.2 \mathrm{fs}$ bears the potential to even resolve ultrafast electronic transitions, in combination with subsequent slower degrees of freedom like atomic motions. These qualities have motivated the design of the Bernina instrument, specialized for studying coupled phenomena in condensed matter by $(a)$ selective manipulation of specific transitions or degrees of freedom and $(b)$ selective probing of the ensuing electronic, magnetic and structural dynamics. We describe here the design and realization of the original concept (Ingold \& Beaud, 2013), as well as the status of commissioning and pilot experiments over a ten-month frame shared with accelerator and Aramis commissioning, installations and developments.

\section{Instrument design}

\subsection{X-ray source}

The linear accelerator of SwissFEL consists of an S-band photoinjector linac and three $\mathrm{C}$-band booster linacs that increase the electron energy to $2.1 \mathrm{GeV}, 3.0 \mathrm{GeV}$ and $5.8 \mathrm{GeV}$, respectively. The nominal normalized slice emittance after the injector is $0.2 \mu \mathrm{m}$ for a bunch charge of $200 \mathrm{pC}$ (Schietinger et al., 2016). A two-stage magnetic bunch compression after the injector $(\mathrm{BC} 1)$ and the first linac $(\mathrm{BC} 2)$ is used to reach a nominal peak current of $3 \mathrm{kA}$. The linac can simultaneously drive two XFELs operated in self-amplified spontaneous emission (SASE) mode at $100 \mathrm{~Hz}$, the hard X-ray Aramis undulator $(0.1-0.7 \mathrm{~nm})$ at $5.8 \mathrm{GeV}$ and the soft X-ray Athos undulator $(0.65-5 \mathrm{~nm})$ at $3.0 \mathrm{GeV}$ (currently under construction). SwissFEL can be tuned from high charge mode ( $200 \mathrm{pC})$ to low charge mode $(10 \mathrm{pC})$, providing between 50 and $5 \mathrm{fs}$ (FWHM) short pulses, respectively. Aramis uses a segmented planar, variable-gap short-period undulator (15 mm, $K=0.1-$ 1.8 ) to generate horizontally linear polarized light (Calvi et al., 2018 ), at $0.1 \%$ bandwidth in normal SASE mode ('pink beam'; presently achieved average bandwidth: $0.4 \%$, Fig. 4). A fourmagnet electron energy collimator before the undulators facilitates two special modes, namely the large-bandwidth mode (relative bandwidth $\sim 4 \%$ ) (Hernandez et al., 2016) and an attosecond mode which exploits full compression of a $10 \mathrm{pC}$ pulse which is expected to produce sub-femtosecond short pulses. Self-seeding at ångstrom wavelengths with increased spectral brightness with respect to SASE is foreseen for the the future (Amann et al., 2012; Prat \& Reiche, 2013). Electron bunch arrival-time monitors (BAMs) with electrooptical detection scheme (Angelovski et al., 2015) are installed after each bunch compressor and at the end of the undulator. They provide non-destructive, shot-to-shot arrival-time information relative to a highly stable pulsed optical reference with resolution better than $5 \mathrm{fs}$ and less than $10 \mathrm{fs}$ drift per day (Arsov et al., 2014). In addition, two C-band transverse deflecting structures are installed at the end of the third linac measuring the longitudinal charge profile and the arrival time of the compressed bunches in two-bunch mode with a resolution of a few femtoseconds.

\subsection{X-ray optics and diagnostics}

The FEL pulses delivered by the Aramis undulator are generally diagnosed non-destructively for intensity and position by a high-transmission ionization chamber with split electrodes. This gas monitor performs over an energy range of 0.025-12 keV at an intensity and position relative (absolute) accuracy of $1 \%(10 \%)$ and about $10 \mu \mathrm{m}$, respectively. A solid state attenuator (silicon and diamond filters) allows the FEL intensity to be reduced. A single-pulse X-ray spectrometer can be inserted in the range 4-12 keV (Rehanek et al., 2017). It uses a thin diamond transmission grating that splits off the first diffraction order in the horizontal plane to illuminate a bent $\mathrm{Si}$ crystal spectrometer. The resolving power $\Delta E / E$ is $10^{-5}-10^{-4}$ in a simultaneously accessible measurement window of $0.5 \%$ bandwidth.

The Aramis optical beamline design enables fast ( $<1 \mathrm{~min})$ switching of the FEL beam between the three experimental stations Alvra, Bernina and Cristallina (Follath et al., 2016). The Bernina beamline propagates straight in the horizontal direction from the Aramis undulator trajectory (Fig. 1). In the vertical direction, the beam path to the experimental station is separated by $20 \mathrm{~mm}$ for radiation safety in a dedicated X-ray 


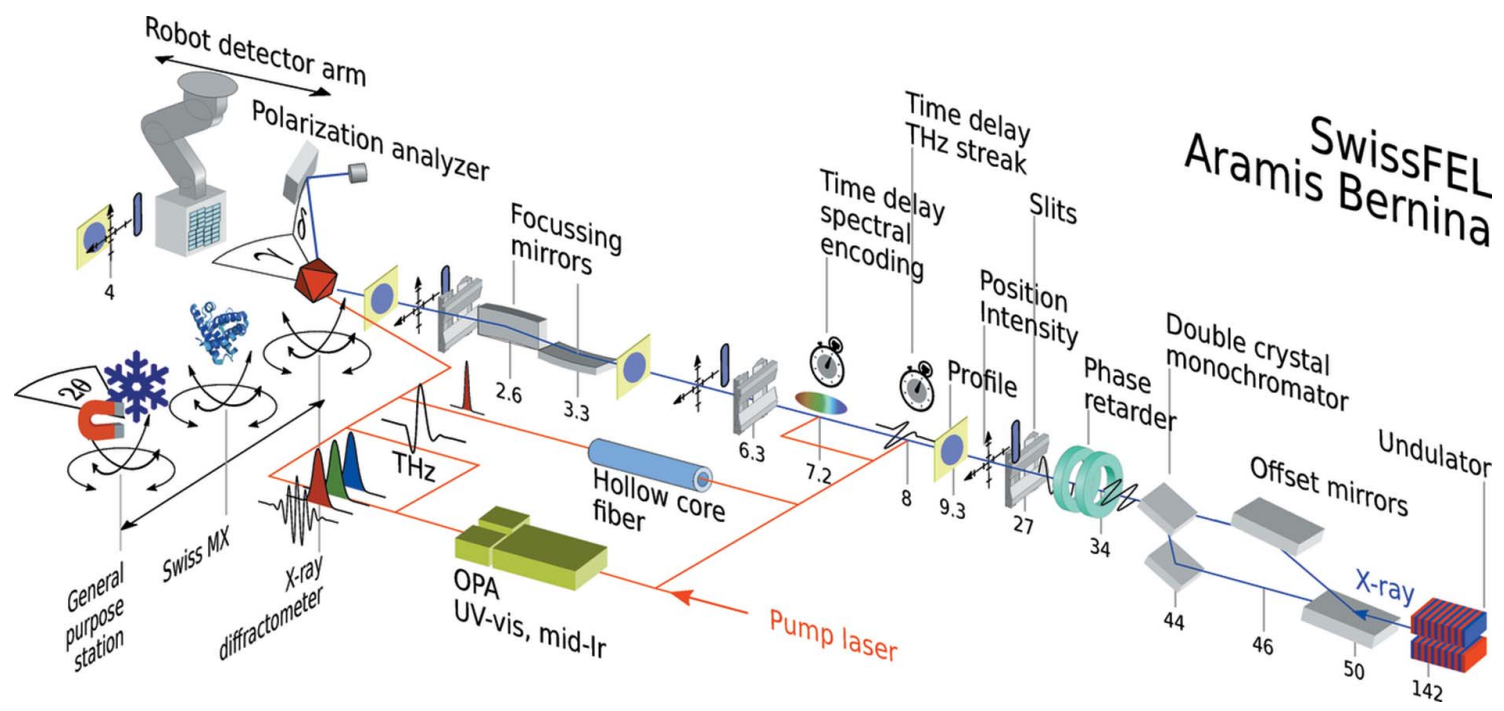

Figure 1

Schematic layout of the Aramis Bernina pump/probe instrument including selected elements. The pump laser source can be converted to different energies and pulse lengths for exciting a specimen. Part of it is used to measure the time delay with respect to the X-ray pulses from the Aramis FEL. The $\mathrm{X}$-ray beamline consists of a set of monochromator crystals as well as beam steering and focusing optics. The X-ray pulse spatial profile, position and intensity are measured at multiple positions in the beamline. The experiments can be carried out at multiple endstations which can be translated into the focused FEL beam.

optics hutch. A vertical deflecting periscope geometry for offset mirrors and monochromator matches the beam paths of the full SASE bandwidth and monochromatic beam, respectively. A pair of bendable plane elliptical offset mirrors (OMs) deflect the beam vertically by $6 \mathrm{mrad}$. Adjustment of curvature allows modest prefocusing in the vertical plane depending on the beam divergence at a given photon wavelength. To prevent single-shot damage by the intense FEL beam and to enhance the reflectance and the critical angle over the full Aramis photon energy range (1.8-12.4 keV), the mirrors are coated with a low- $Z\left(10 \mathrm{~nm} \mathrm{~B}_{4} \mathrm{C}\right.$ on top of $\left.36 \mathrm{~nm} \mathrm{SiC}\right)$ and mid- $Z$ (15 nm $\mathrm{B}_{4} \mathrm{C}$ on top of $20 \mathrm{~nm} \mathrm{Mo}$ ) bilayer leaving a blank area of uncoated silicon between them. Uncoated silicon has the lowest critical energy of about $10 \mathrm{keV}$ and may be used at low energies when harmonic rejection above $3.5 \mathrm{keV}$ becomes important. Switching from pink to monochromatic mode is accomplished by $\mathrm{OM}$ retraction and insertion of the double-crystal monochromator (DCM). The DCM has three crystal pairs mounted on common Braggrotation axes of incidence angular range $5-70^{\circ} . \mathrm{Si}(111)$ and $\mathrm{Si}(311)$ crystals can be used for standard and high-resolution applications (calibration example in Fig. 4). An additional pair of $\mathrm{InSb}(111)$ crystals extends the photon energy range to tender X-rays down to $1.8 \mathrm{keV}$. The DCM geometry with translation perpendicular to the second crystal surface enables an adjustable beam offset up to $32 \mathrm{~mm}$. An installation of additional harmonic rejection mirrors, foreseen in the original design, has received lower priority, based on first measurements in the tender X-ray regime where the focusing mirrors (see below) sufficiently rejected the higher harmonics.

Behind the DCM, a diamond phase retarder is foreseen in order to control the X-ray polarization. The option to create and control circular polarization will increase the sensitivity and selectivity in resonant X-ray diffraction (RXRD) to magnetic and oriented electronic structures. The rotation of the linear polarization plane also facilitates studying oriented structures at identical pump/probe geometry in time-resolved resonant X-ray diffraction (tr-RXRD). Operated in Bragg or Laue transmission geometry a polarization of $80-95 \%$ has been achieved above $7 \mathrm{keV}$ at synchrotron beamlines (Strempfer et al., 2013) and at XFELs (Suzuki et al., 2014). A double-stage phase retarder with two crystals operated in series allows to compensate for depolarizing effects due to angular divergence and energy spread (Scagnoli et al., 2009; Francoual et al., 2013) and also achieve higher degrees of linear polarization. Undulator-based polarization control (Schmidt \& Calvi, 2018) in an 'afterburner' configuration (Lutman et al., 2016) is investigated for future upgrade.

Before transportation of the FEL beam from the optics hutch to the Bernina experimental station, the X-ray beam trajectory, profile and intensity can be measured and aligned by multiple beam-defining apertures (four-blade slits), profile monitors (based on Ce:YAG scintillators) and quadrant detection intensity and position monitors (Tono et al., 2011). They allow well defined conditions to be reproduced before and after deflection from X-ray optics.

Pulse-to-pulse arrival-time jitter between the X-ray pulses and the optical laser may severely limit the temporal resolution available for time-resolved experiments. Different methods are currently commissioned that measure the relative laser pump and X-ray arrival time at different locations for every pulse. The separate arrival time of FEL electron bunches and pump laser pulses can be measured by the BAM at the end of the undulator line and a laser arrival monitor (LAM) with respect to a master reference clock at an expected overall time resolution of around $50 \mathrm{fs}$ FWHM. Improved and more representative temporal resolution can be reached by direct cross-correlation of FEL X-rays and optical laser pulses 
within the beamline and near the experiment. This is achieved by a streaking of X-ray pulse generated photoelectrons by a $\mathrm{THz}$ electric field generated from the pump laser source (Gorgisyan et al., 2017). Thanks to a gas target, the method can be used over the entire X-ray photon energy range at high X-ray transmission. An X-ray time resolution of $\leq 40 \mathrm{fs}$ FWHM is expected. A relative cross-correlation resolution down to a few femtoseconds can be reached by the spectral encoding timing diagnostics (Hartmann et al., 2014; Harmand et al., 2013; Bionta et al., 2011). As the performance in this case depends highly on sufficient X-ray intensity, a location close to the X-ray focus ( $1 \mathrm{~m}$ from sample location) can be used additionally to the built-in device indicated in Fig. 1.

A pair of focusing mirrors in orthogonal Kirkpatrick-Baez (KB) geometry are installed at $2.6 \mathrm{~m}$ working distance upstream of the sample position. They combine achromaticity with high transmission over a large energy range (Yumoto et al., 2013). The mirrors $\left(\mathrm{B}_{4} \mathrm{C} /\right.$ Mo-coating, $500 \mathrm{~mm}$ length $)$ operate at grazing incidence angle, which can be chosen between 4 and $12 \mathrm{mrad}$ total deflection. This allows suppression of higher harmonics from the SASE beam by a factor of $10^{-3}$ to $10^{-4}$. With mirror benders and a profile error of $\leq 2 \mathrm{~nm}$ in the mirror centre, the spot at the sample location can be adjusted between 2 and $\sim 300 \mu \mathrm{m}$ FWHM, independently in the horizontal and vertical directions (Fig. 2).

In order to control and reproduce the beam pointing of the KB-focused FEL beam, additional profile and intensity/position monitors are installed upstream and downstream with respect to the sample position. They are mounted on highprecision motorized tables, a six degrees of freedom heavyload hexapod holding the upstream diagnostics and a two-axis translation table supporting the downstream diagnostic devices. Those allow the diagnostics to be positioned at identical positions relative to the FEL beam, also upon change

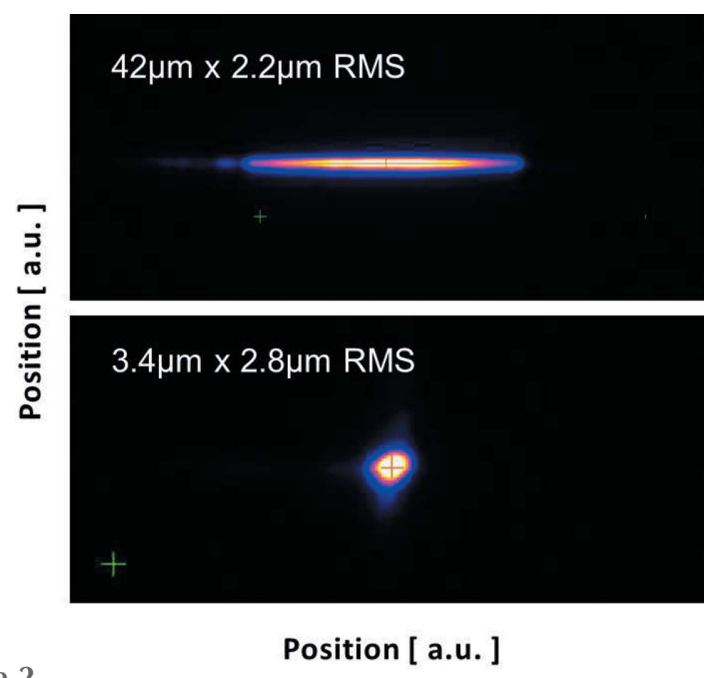

Figure 2

Focal spot for a line and point focus at $6.6 \mathrm{keV}$ (third harmonic) measured at the Bernina sample position during early commissioning (fundamental FEL energy at $2.2 \mathrm{keV}$ ). The focal spot was measured in air with a profile monitor after transmission through a diamond $(50 \mu \mathrm{m})$ window separating the beamline vacuum system from the experimental area.
Table 2

Laser options at the SwissFEL Bernina instrument.

\begin{tabular}{|c|c|}
\hline $\begin{array}{l}\text { Experimental branch } \\
\text { Short pulse option }\end{array}$ & $\begin{array}{l}30 \mathrm{fs} \dagger, 10 \mathrm{~mJ}, 800 \mathrm{~nm} \\
<10 \mathrm{fs}, 300 \mu \mathrm{J}\end{array}$ \\
\hline \multicolumn{2}{|c|}{ HE-TOPAS $\$$ (pump: $10 \mathrm{~mJ}, 30 \mathrm{fs}$ ) } \\
\hline Signal and idler & $1160-2600 \mathrm{~nm} \S$ \\
\hline VIS-UV extensions & $240-1160 \mathrm{~nm} \S$ \\
\hline DFG extensions & $2600-15000 \mathrm{~nm} \S$ \\
\hline \multicolumn{2}{|c|}{ TOPAS twins $\ddagger$ (pump: $10 \mathrm{~mJ}, 100 \mathrm{fs}$ ) } \\
\hline DFG extensions & $2600-20000 \mathrm{~nm} \S \Phi$ \\
\hline
\end{tabular}

$\dagger$ Option 100 fs. \$ For details see http://lightcon.com/Products/opa-topas.html. $\S$ Pulse energies and durations on best effort basis. - CEP-stable.

of the KB grazing angle. The upstream diagnostic chamber houses an optional arrival-time monitor, an X-ray profile and intensity monitor, as well two slit systems to remove beam halo.

\subsection{Timing synchronization and optical laser}

A global timing reference for all timing-sensitive components at SwissFEL is generated by an optical master oscillator. For optimized overall phase noise performance it is synchronized to an RF master oscillator which itself is locked to a $10 \mathrm{MHz}$ rubidium frequency standard. The integrated absolute jitter from $10 \mathrm{~Hz}$ to $1 \mathrm{MHz}$ is as low as $50 \mathrm{fs}$ FWHM (Milne et al., 2017). The most critical clients of SwissFEL require $<35$ fs FWHM jitter and down to 10 fs peak-to-peak temporal drift per day (Schietinger et al., 2016). Critical clients such as the experimental laser system, the BAM and LAM monitors, are fibre-coupled to pulsed optical links.

The laser system (Erny \& Hauri, 2016) for the experimental stations consists of two identical commercial $100 \mathrm{~Hz}$ Ti:sapphire amplifier systems with a compressed output energy of $20 \mathrm{~mJ}$ at $800 \mathrm{~nm}$. These lasers are installed above the experimental stations in a separate clean room with air $\left( \pm 0.1^{\circ} \mathrm{C}\right)$ and humidity (40-45\%) control. To ensure simultaneous and independent operation of the experimental stations both lasers can serve either station. The uncompressed amplifier output is sent through an evacuated transfer line to optical tables in the Bernina hutch where it is split into two branches, one for the experiment and the other for timing diagnostics. The diagnostic branch is used to operate the $\mathrm{THz}$ arrival-time monitor for which the $\mathrm{THz}$ electrical field is generated using the tilted pulse front scheme in $\mathrm{LiNbO}_{3}$ (Hebling et al., 2002).

The experimental branch includes the LAM to measure the laser arrival near the experiment and non-linear conversion stages offering a broad range of pump frequencies. The LAM is based on a spectrally resolved cross-correlation with a resolution of 12 fs FWHM (Csatari Divall et al., 2015). For experimental use, a range of parameters can be derived from the compressed output of $10 \mathrm{~mJ},<30 \mathrm{fs}, 800 \mathrm{~nm}$ (Table 2). A short-pulse option offers $<10$ fs pulses with $>200 \mu \mathrm{J}$ at $800 \mathrm{~nm}$ generated by spectral broadening via self-phase modulation in a gas-filled hollow core fibre and subsequent pulse compression (Nisoli et al., 1996). Frequency tuning is achieved via an optical parametric amplifier (OPA), combined with 
subsequent frequency mixing stages covering a broad wavelength range from the UV up to the mid-IR $(0.24-15 \mu \mathrm{m})$. The signal branch of the OPA output can also be used to generate intense single-cycle $\mathrm{THz}$ pulses by optical rectification of near-IR pulses in organic non-linear crystals (DAST, OH1, DSTMS) (Hauri et al., 2011; Ruchert et al., 2013; Kozina et al., $2017 b$ ) or by the tilted pulse front technique in $\mathrm{LiNbO}_{3}$ (Hebling et al., 2002; Kozina et al., 2017a), yielding field strengths up to a few hundreds of $\mathrm{kV} \mathrm{cm}^{-1}$ in the frequency range $0.3-3 \mathrm{THz}$.

Single-cycle THz pulses can be used to excite (resonantly or non-resonantly) large-amplitude atomic motions with electric and magnetic fields exceeding $1 \mathrm{MV} \mathrm{cm}^{-1}$ and $1 \mathrm{~T}$, respectively. Single-cycle $\mathrm{THz}$ pulses are intrinsically stable with respect to their carrier-envelope phase (CEP) and the optical cycle is long compared with the FEL pulse duration, allowing the field-induced dynamics to be tracked. So far mostly resonant field driven excitations in the $0.3-2 \mathrm{THz}$ range probed by FEL X-ray diffraction have been applied to study soft mode dynamics in the prototypical ferrolectrics $\mathrm{Sn}_{2} \mathrm{P}_{2} \mathrm{~S}_{6}$ (Grübel et al., 2016), $\mathrm{BaTiO}_{3}\left(\right.$ Chen et al., 2016) and $\mathrm{SrTiO}_{3}$ (Kozina et al., 2017a,b), the magneto-electric excitation of the spin cycloid in the multiferroic $\mathrm{TbMnO}_{3}$ (Kubacka et al., 2014) and the initiation of the metal-to-insulator transition in $\mathrm{VO}_{2}$ (Gray et al., 2018).

By exploiting selective excitation and control of higherenergy modes in the frequency range $15-20 \mathrm{THz}(20-15 \mu \mathrm{m})$, recent experiments have demonstrated efficient non-linear coupling from the resonantly excited IR-active mode to Raman modes along the symmetry breaking coordinate (Först et al., 2011a). The suppression of magnetic and orbital order (Först et al., 2011b, 2015), enhanced superconductivity in high$T_{\mathrm{c}}$ superconductors (Mankowsky et al., 2014; Mitrano et al., 2016; Fausti et al., 2011) or ultrafast switching of the ferroelectric polarization (Mankowsky et al., 2017; von Hoegen et al., 2018) has been demonstrated. In one case non-linear electron-phonon coupling was found to dominate directly the charge order melting in a doped manganite (Esposito et al., 2017).

With the potential to reach sub-10 fs time resolution, fieldinduced dynamics at mid-IR frequency can be studied with CEP-stable narrow-bandwidth and frequency-tunable pulses. For that purpose a CEP-stable system based on two independent OPAs (TOPAS-twins, Light Conversion) is being installed at Bernina. Developments that extend the available tuning range to the so-called $\mathrm{THz}$ gap in the range $4-20 \mathrm{THz}$ $(15-75 \mu \mathrm{m})$ are closely followed for potential future implementation (Liu et al., 2017; Cartella et al., 2017).

The large range of laser parameters available for experiments at Bernina requires flexible incoupling with respect to the X-ray beamline. Especially for mid-IR/THz wavelengths, flexible mounting provisions for optical support tables and breadboards are provided close to the sample location in order to facilitate use of small-focal-length focusing optics. For UV/VIS and IR wavelengths, exchangeable dedicated incoupling vacuum chambers for high vacuum and intermediate/ helium atmosphere are mounted on the heavy-load upstream diagnostics hexapod table between the diagnostics element and the experimental endstations. They are separated by an $\mathrm{X}$-ray window $(50 \mu \mathrm{m}$ CVD diamond) from the diagnostics chamber which is retractable for windowless operation.

\subsection{Endstations}

The endstation design (Ingold et al., 2016) emphasizes rapid reconfiguration capability in terms of sample environment, spectrometers and detector geometries to support a wide variety of experiments. For this purpose two endstations for X-ray pump-probe (XPP) experiments are installed, a general purpose station (Bernina-GPS) as well as a six-circle X-ray diffractometer (Bernina-XRD). Both endstations are mounted on a high-precision rail system oriented perpendicular to the X-ray trajectory, allowing them to be exchanged and reproducibly positioned at the sample location (Fig. 1). Heavy-load capability allows the stations to support and precisely align a variety of custom sample stage modules such as goniometers and sample environmental setups which can be swapped and mounted on both Bernina-GPS and BerninaXRD. Sample modules provided by an experimental team or user group may include cryogenic vacuum chambers, coldfinger cryostats, superconducting high-field magnets or cryogenically cooled pulsed magnet systems and high-pressure diamond anvil cells. As a third station which is mounted temporarily for periods of time on the same rail system, a fixed-target protein crystallography station (SwissMX) is operated at Bernina (Fig. 3).

The Bernina-GPS station uses a high-precision positioning platform that can be aligned with respect to the FEL beam trajectory in three degrees of freedom compensating for pointing changes induced by the KB focusing system. This platform supports two concentric, vertical rotation axis circle stages for sample environment and detector systems. The sample circle can be combined with sample diffractometer modules; the detector circle can support heavier detectors or analyzers $(\leq 300 \mathrm{~kg})$ at about $1 \mathrm{~m}$ distance from the sample interaction.

The Bernina-XRD station platform is identical to the Bernina-GPS station except for the detector circle configuration. The detector arm has two rotation circles, with a vertical and a horizontal rotation axis. It supports two motorized translation detector stages carrying a $1.5 \mathrm{M}$ Jungfrau area detector and a vacuum polarization analyzer stage with angular collimation and point detector, respectively (Strempfer et al., 2013). The stages are offset by $20^{\circ}$ to allow rapid switching between the analyzer stage and the Jungfrau detector. The sample-detector distance of $790 \mathrm{~mm}$ and pixel size of $75 \mu \mathrm{m} \times 75 \mu \mathrm{m}$ provides an angular resolution of $0.00547^{\circ}\left(\Delta Q=5.4 \times 10^{-4}\right.$ at $\left.11.2 \mathrm{keV}\right)$.

On the single sample circles of both endstations an additional choice of three pre-configured sample diffractometer stages can be mounted. They support different motion degrees of freedom and ranges for sample environment of different size and weight. A $\kappa$-goniometer with ascending motorized horizontal sample axis, $\kappa$ axis and additional six degrees of 


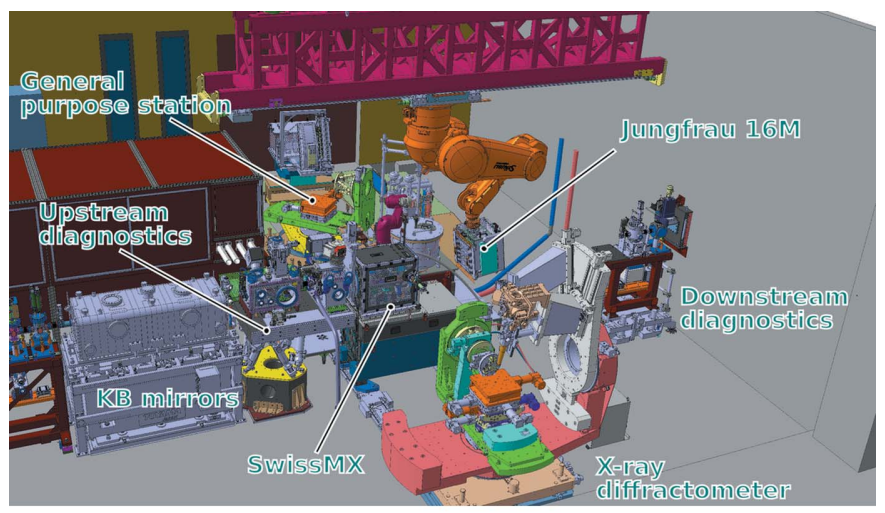

(a)

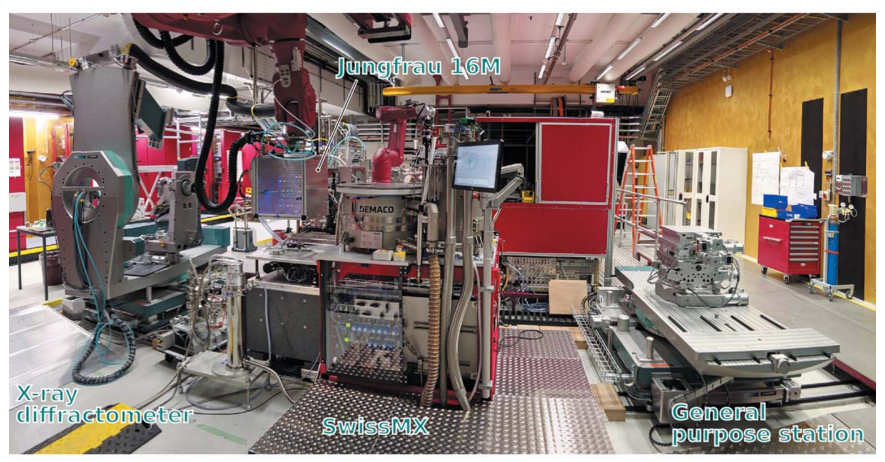

(b)

Figure 3

Experimental area and endstations. Top: CAD-view showing the robot detector mount and the three experimental endstations. Bottom: photograph in the direction upstream of X-ray propagation.

freedom is used to position sample systems of up to $\sim 0.3 \mathrm{~kg}$ weight. This leaves space above the sample and on the free side so that, by suitable combination of rotations, a wide range of reciprocal space can be accessed without parts of the diffractometer obstructing either the incident or scattered $\mathrm{X}$-ray beams or the optical pump. For intermediate sample environments like small vacuum chambers, a hexapod goniometer consisting of a hexapod mounted on a horizontal rotation axis can be used. For sample environments like large sample chambers, cryostats or superconducting magnets, a non-magnetic heavy-load five-degrees-of-freedom goniometer (load capacity of $700 \mathrm{~kg}$ ) can be used on both endstations.

The detection stages for both endstations can be combined with a ceiling-mounted robot detector arm, which normally holds a large-area Jungfrau $16 \mathrm{M}$ pixel detector. The sampledetector distance can be varied along the beam axis by a translation stage holding the entire robot arm, for example, to perform SAXS measurements (Kim et al., 2017) with momentum resolution $\Delta Q=1.2 \times 10^{-4} \AA^{-1}(10 \mathrm{keV})$ at a maximum distance of $3.1 \mathrm{~m}$. It can further be used for coherent Bragg diffraction on nano- and micro-crystalline materials (Clark et al., 2013), time-domain measurements of density fluctuations by diffuse scattering (Trigo et al., 2013), and combined X-ray emission (XES) and diffraction experiments to resolve the electronic and geometric structure of catalytic and macromolecular micrometre-sized crystals (Kern et al., 2013).
The Bernina-XRD endstation is intended particularly for sample environments at extreme temperatures, pressures or magnetic fields which facilitates different classes of experiments: suppression of stripe order (Fausti et al., 2011) or enhancement of superconductiviy far above $T_{\mathrm{c}}$ ( $\mathrm{Hu}$ et al., 2014; Mankowsky et al., 2014) in high- $T_{\mathrm{c}}$ materials, the competition of CDW and superconducting phases at low temperatures $(T \leq 10 \mathrm{~K})$ and high magnetic fields $(H \geq 30 \mathrm{~T})$ (Jang et al., 2016; Chang et al., 2012, 2016; Gerber et al., 2015), or pressure $(P \leq 20 \mathrm{GPa})$ driven quantum critical behaviour in 2D magnetic systems (Jaramillo et al., 2009; Haravifard et al., 2012; Feng et al., 2012), and iron-based superconductivity (Takahashi et al., 2015).

The heavy-load detector arm of Bernina-GPS allows experiments with large emission spectrometers as used for resonant inelastic X-ray scattering (RIXS), which has been demonstrated as time- and momentum-resolved hard X-ray RIXS (tr-qRIXS) at an XFEL (Dean et al., 2016). A compact five-analyzer RIXS spectrometer $(R=1 \mathrm{~m})$ (Moretti Sala et al., 2018) will be available at Bernina-GPS to study the dynamics of charge and $d d$-excitations in $3 d$ transition metal oxides ( $K$-edge XES/RIXS), and dispersive spin-exciton and magnon excitations in $5 d$ systems ( $L$-edge RIXS) that exhibit strong spin-orbit coupling (SOC). For measurements at a well defined momentum in $q$-space only the central analyzer will be used. Spherical bent diced analyzers (Gog et al., 2013) are operated on a Rowland circle such that the sample, central analyzer and detector are all mounted on a circle of radius $R / 2$ (Johan geometry). The X-rays backscattered from the analyzer are detected with a $250 \mathrm{~K} 2 \mathrm{D}$ Jungfrau detector with pixel size of $225 \mu \mathrm{m}(\mathrm{h}) \times 25 \mu \mathrm{m}(\mathrm{v})$. The overall resolution at $11.2 \mathrm{keV}$ (Ir $L_{3}$-edge) is $50-70 \mathrm{meV}$ for an $\mathrm{Si}(444) \mathrm{DCM}$ and $\mathrm{Si}(844)$ diced-crystal analyzer. Adopting a horizontal scattering plane and dispersion in the vertical plane complies well with an asymmetric X-ray footprint (vertical focus) on the sample to preserve energy- and time-resolution when matching of the penetration depth of the laser-pump and $\mathrm{X}$-ray-probe is required at grazing incidence $\theta \simeq 0.5-5^{\circ}$ (Johnson et al., 2008, 2010). Tr-qRIXS requires high spectral flux and would greatly benefit from self-seeding operation (Amann et al., 2012) of Aramis in the future to enhance the spectral brightness by a factor of 5-8.

The Bernina-XRD station is intended to enable experiments that require precise reciprocal space measurements. Besides time-resolved resonant and non-resonant XRD, it additionally allows measurements of polarization-resolved trRXRD. Time-resolved diffraction experiments on correlated systems such as mixed-valence CMR maganites (Beaud et al., 2009 , 2014) can be extended to selectively probe different components of the resonant scattering tensor by measuring the polarization dependence in the presence of cooperative oriental alignment caused by competition between charge- or orbital-ordered phases and magnetic phases. This is done by resolving the scattering into the respective $\sigma-\sigma^{\prime}$ and $\sigma-\pi^{\prime}$ polarization channels where $\sigma(\pi)$ and $\sigma^{\prime}\left(\pi^{\prime}\right)$ denote the polarization perpendicular (parallel) to the scattering plane for the X-rays in the entrance and exit channel, respectively 
(Zimmermann et al., 2001; Grenier et al., 2004). From the intensities of the $\sigma$ - and $\pi$-components the Poincaré-Stokes parameters $P_{1}$ and $P_{2}$ for the linear polarization state are derived and the degree of circular polarization $P_{3}$ is inferred from $P_{1}^{2}+P_{2}^{2}+P_{3}^{2} \leq 1$ (Paolasini et al., 2007; Mazzoli et al., 2007).

In magnetic systems the transient magnetic order due to $4 f-5 d$ magnetic coupling (Rettig et al., 2016) or frustrated exchange interactions (Johnson et al., 2012, 2015) could be resolved. Similarly the magnetic and orbital ordering in a bilayer perovskite ruthenate (Bohnenbuck et al., 2008) and the magnetic structure in a bilayer SOC iridate (Kim et al., 2012) have been determined. In a combined RXRD and RIXS study the unresolved magnetic ground state in a prototypical pyrochlore iridate has been clarified (Donnerer et al., 2016). In some cases polarization analyzer scans are combined with scans of the azimuthal angle where the sample is rotated around the scattering vector. These combined scans directly measure the symmetries of the tensors involved in resonant scattering amplitudes. However, pump-probe experiments impose geometric constraints and azimuthal scans will be of limited use in practice. Instead the use of diamond phase retarders in combination with a polarization analyzer would be the preferred solution for polarization-resolved tr-RXRD measurements.

Fixed-target serial femtosecond crystallography (FT-FX) (Hirata et al., 2014; Feld et al., 2015; Roedig et al., 2015, 2016; Opara et al., 2017) is an additional activity at Bernina. The SwissMX endstation (Pedrini et al., 2017) is installed at Bernina from time to time for FT-FX experiments. The main element of the station is a sample diffractometer placed inside an experimental chamber suitable for various data collection schemes which require precise positioning of the protein samples when hit by the femtosecond X-ray pulses. The station also includes a cryo-jet, room-temperature sample reservoirs, a liquid-nitrogen sample reservoir for up to 500 sample pins, as well as a dedicated robot for automated sample exchange. Both serial scanning data collection (FT-SFX) at $100 \mathrm{~Hz}$ from $3 \mathrm{D}$ micro-crystals of size $<5 \mu \mathrm{m}$ positioned on a chip as well as synchrotron-like data collection of larger crystals by rotation are foreseen. The measurements can be performed in air or in helium atmosphere with X-rays as low as $5 \mathrm{keV}$. During data collection the samples are maintained either at room temperature or at cryogenic temperatures using a nitrogen or helium gas stream cryocooler. The diffraction images from the protein crystals will be collected with the $16 \mathrm{M}$ Jungfrau detector mounted on the robot detector arm. By positioning the detector immediately downstream of the experimental chamber a resolution of better than $1 \AA$ can be achieved with $12 \mathrm{keV}$ radiation.

\subsection{Detector and data acquisition}

The Jungfrau pixel detector has specifically been developed for the needs of the Aramis endstations (Mozzanica et al., 2014, 2018) by the detector group at PSI. It is a charge integrating detector using a hybrid architecture consisting of silicon sensors bump-bonded with a readout ASIC (application specific integrating circuit). The standard Jungfrau ASIC consists of $256 \times 256$ pixels, each pixel being $75 \mu \mathrm{m} \times 75 \mu \mathrm{m}$ in size. Each pixel incorporates a preamplifier with three gains, a switching block that enables an automatic gain switching pixel by pixel, a sampling stage consisting of a storage array for 16 images, and a readout buffer. The output of the ASIC is a signal proportional to the number of photons interacting with the Si sensor. Arrays of $2 \times 4$ chips $(512 \times 1024$ pixels $)$ form a module of about 0.5 Mpixel. The readout board uses a FPGA to generate control signals for the readout ASICs and receives the data stream from the ADCs (14 bit) which digitize the multiplexed analogue output from the chips. The readout time set by the ADC clock speed (40 MHz) corresponds to a frame rate of $2.5 \mathrm{kHz}$.

Experiments at Bernina will profit from several features of the Jungfrau detector. Firstly, the noise level, well below Poisson statistical fluctuations across the full dynamic range, is low enough at the highest gain to allow single-photon detection even at tender X-ray energies in the range $2-4 \mathrm{keV}$. Secondly, automatic gain switching with three levels of gain for individual pixels raises the overall dynamic range of the detector to $10^{4}$ per pixel per pulse. This is particulary important for simultaneous measurement of diffuse scattering or Bragg diffraction from charge-, orbital- or spin order together with up to 5000 times more intense Bragg reflections. Thirdly, the energy resolution capability (see Fig. 4) allows efficient discrimination of elastic from fluorescence signals at lower count rates, which can be used to, for example, suppress background signals. Special use-oriented variations of the sensor design have been developed, like the high spatial resolution in one dimension (pixel size $25 \mu \mathrm{m} \times 225 \mu \mathrm{m}$ ) for spectrometer applications. For in-vacuum operation the sensors and detector chips can be mounted in vacuum $\left(10^{-6} \mathrm{mbar}\right)$, cooled by a cooling plate $(\sim 20 \mathrm{~W}$ per module $)$ and separated by a vacuum flange from the readout board which is operated in air.

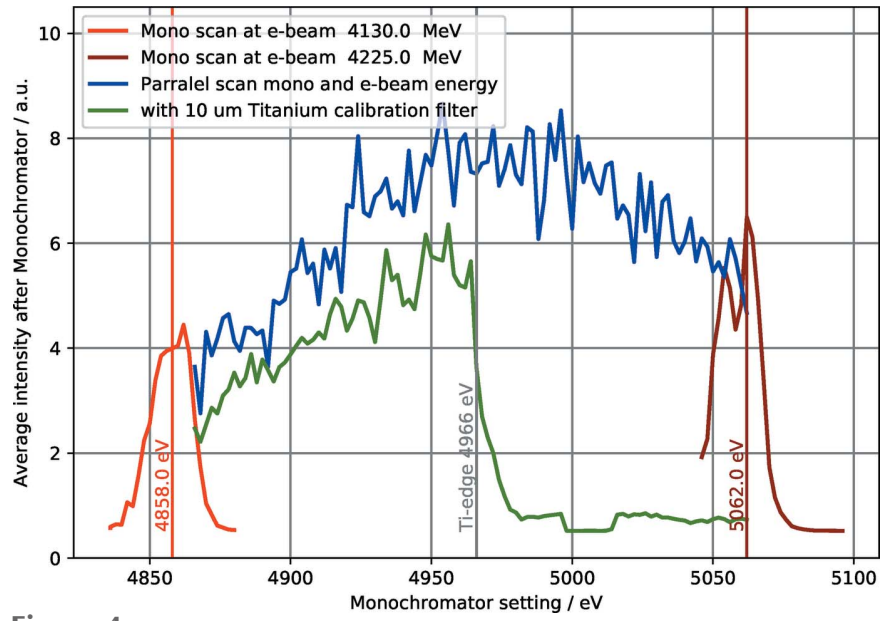

Figure 4

SwissFEL Aramis average bandwidth measurement (for two different electron energy settings). The monochromator energy can be adjusted in parallel to the electron energy in order to scan a larger energy range, e.g. for calibration purpose. 
SwissFEL uses a de-centralized data acquisition system recording data asynchronous and synchronous to the individual FEL pulses. Asynchronous data are predominantly based on the channel access protocol EPICS. Synchronous data are based on ZeroMQ streams which are tagged by Id numbers for each FEL pulse (pulse-Ids) from their data source. This allows scalable operation of even multiple large data sources like the $16 \mathrm{M}$ Jungfrau detector, through separate individual data streams to disk storage and recombination of data from individual pulses by data buffering and pulse-Id based data access. Experimental data are stored to multiple data files (hdf5 format) by a system of separated data acquisition systems for experimental data from Jungfrau detectors, 2D camera images, scalar and 1D waveform data as well as asynchronous data. The systems match the individually saved data ranges by communicating the requested pulse-Ids and data time-stamps. 2D camera images, scalar and 1D waveform data and asynchronous data are in addition permanently buffered for diagnostics purposes over hours or days.

Combined manual and programmatic control of the various subsystems is possible by a high-level scripting framework of Python libraries. It can be used standalone or as a library for specialized applications. It includes standard data acquisition functionality like step-by-step and continuous scans, and can access acquired data for automatization of procedures. A distributed online data processing framework is under development.

\subsection{Commissioning and first experiments}

The Aramis undulator has been in first operation since the end of 2016, and has been commissioned step by step within the following year with interruptions for installations.

The first pilot experiment at SwissFEL was performed in November 2017 at the Bernina instrument after several weeks of commissioning of prioritized elements in the X-ray beamline. A light-induced semiconductor-to-metal transition in titanium pentaoxide $\mathrm{Ti}_{3} \mathrm{O}_{5}$ was measured at Bernina by femtosecond grazing-incidence powder diffraction (M. Cammarata, Rennes University). The saturated SASE pulse energy was $200 \mu \mathrm{J}$ at $2.20 \mathrm{keV}(K=1.2)$ of the fundamental at electron energy $2.38 \mathrm{GeV}$, bunch charge $205 \mathrm{pC}$ and repetition rate $10 \mathrm{~Hz}$. The experiment was performed using pink X-ray beam at $6.6 \mathrm{keV}$ and $0.1 \%$ bandwidth using the third harmonic of the fundamental. The instrumental time resolution of about $350 \mathrm{fs}$ FWHM was measured by light
$(800 \mathrm{~nm})$ induced changes in the diffracted $\mathrm{Bi}(111)$ intensity from a $40 \mathrm{~nm}$ bismuth expitaxial thin film of $40 \mathrm{~nm}$ (Johnson $e t$ al., 2008). The time resolution was mainly limited by electron bunch compression and pump laser synchronization. No timing diagnostics for time jitter and drift corrections were applied. X-ray powder diffraction patterns from a polished nanoparticle pellet of 20-30 nm-sized crystallites were recorded with a $1.5 \mathrm{M}$ Jungfrau pixel detector. The energy resolution of the detector allowed gating to suppress background due to Ti $K \alpha$ fluorescent photons (Fig. 5). The pump fluence at $800 \mathrm{~nm}$ and spot size $300 \mu \mathrm{m} \times 300 \mu \mathrm{m}$ FWHM was varied in the range $0.16-0.44 \mathrm{~mJ}$. The focal spot size of the X-ray was $100 \mu \mathrm{m}(\mathrm{h}) \times 5 \mu \mathrm{m}(\mathrm{v})$ FWHM and the incidence angle was varied in the range $0.3-0.5^{\circ}$ to probe the phase transition as a function of depth at the nanoscale. The timeresolved powder patterns shown in Fig. 6 hold information on the volume and crystalline phase fractions as a function of time. The metallic phase has a higher unit cell volume and thus the semiconductor-to-metal transition may occur only after pressure relaxation. To clarify this the time resolution of $350 \mathrm{fs}$ FWHM is sufficient because volumetric changes of $\sim 25 \mathrm{~nm}$ sized crystallites proceed on the acoustic timescale of $\sim 0.5 \mathrm{ps} \mathrm{nm}^{-1}$.

The second pilot experiment was performed in March 2018. Single-cycle $\mathrm{THz}$ pulses were used to directly drive lattice vibrations in the ferroelectric (FE) model compound $\mathrm{Sn}_{2} \mathrm{P}_{2} \mathrm{~S}_{6}$ with a time-dependent electric field (M. Savoini and $\mathrm{S}$. Johnson, ETH Zurich). Lowering the temperature below 

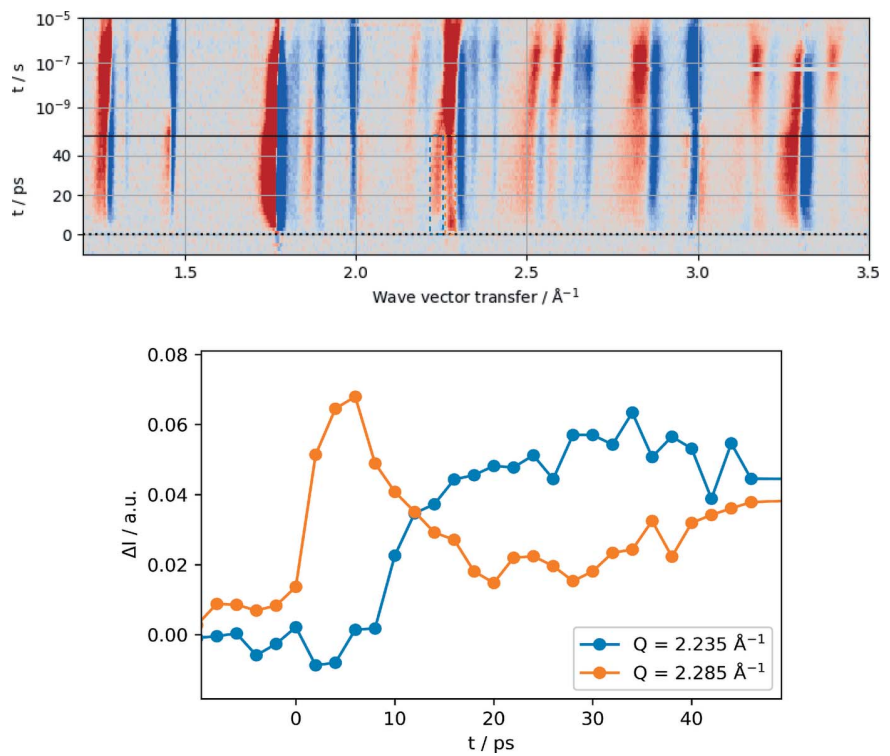

Figure 6

Bernina first pilot experiment. Top: light-induced differential scattering pattern collected from $\mathrm{Ti}_{3} \mathrm{O}_{5}$ nanocrystals in Debye-Scherrer geometry as a function of momentum transfer and time delay. The powder patterns have been collected with a 2D 1.5M Jungfrau pixel detector. Bottom: relative change of diffraction intensity as a function of time delay between the $800 \mathrm{~nm}$ pump and X-ray probe at $6.6 \mathrm{keV}$. For two momentum transfers indicated by the dashed box in the top panel a distinct different time behaviour is observed at shorter times. The instrumental time resolution derived from $\mathrm{Bi}(111)$ diffraction intensity prior to the experiment was about $350 \mathrm{fs}$ FWHM.

$337 \mathrm{~K}$ induces a soft-mode driven displacive phase transition to a ferroelectric state. The structural changes associated with the paraelectric-to-ferroelectric phase transition can mainly be attributed to a displacement of the $\mathrm{Sn}^{2+}$ ion and its direction roughly coincides with the direction of the polarization in the ferroelectric phase. The displacement can also directly be linked to a soft phonon mode near $1 \mathrm{THz}$ at room temperature. By exciting this soft phonon mode with broadband $\mathrm{THz}$ pulses the structural modulation has been measured directly and quantitatively in a recent tr-XRD experiment (Grübel et al., 2016) at the FEMTO slicing facility at the SLS (Beaud $e t$ al., 2007; Ingold et al., 2008). In a subsequent experiment at the X-ray pump-probe instrument (XPP) at the LCLS (Chollet et al., 2015), additional modes by using higher THz fields have been observed. In continuation of these experiments the pilot experiment was designed to identify and quantify dissipation mechanisms in the strongly non-equilibrium state that possibly impact the feasibility of FE switching.

The experiment was performed with monochromatic X-rays at $6.0 \mathrm{keV}$ using the third harmonic at $0.015 \%$ bandwidth. Aramis was operated at $2.5 \mathrm{GeV}$ and bunch charge $200 \mathrm{pC}$ at $10 \mathrm{~Hz}$ to reach saturation at $2.0 \mathrm{keV}(K=1.45)$ of the fundamental. The transverse deflecting structures (TDS) installed prior to the second pilot experiment allowed good control of the bunch length by the two bunch compressors $\mathrm{BC} 1$ and $\mathrm{BC} 2$. The saturated pulse energy (pulse length) of the SASE pulses was $205 \mu \mathrm{J}$ (90 fs FWHM) and $350 \mu \mathrm{J}$ (170 fs FWHM) depending on compressor settings. The electron beam arrival-time jitter measured with TDS was about $70 \mathrm{fs}$

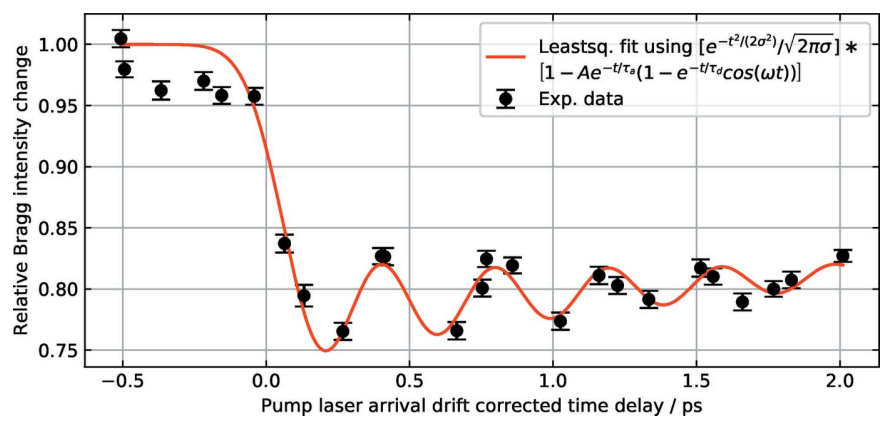

Figure 7

Jitter-limited time resolution. Laser $(800 \mathrm{~nm})$ induced coherent $A_{1 g}$ phonon mode in a $40 \mathrm{~nm}$ thin (111)-oriented film of crystalline bismuth at an incident fluence of $1 \mathrm{~mJ} \mathrm{~cm}^{-2}$. The fit by the model function in the plot legend reveals a time resolution of $110 \mathrm{fs} \sigma$ (r.m.s.). Timing-drifts of the pump laser timing were corrected for by the laser arrival monitor (LAM).

and $90 \mathrm{fs}$ FWHM, respectively. The shot-to-shot jitter measured independently with a BAM installed after BC1 was in agreement with the TDS measurements. The timing drift measured with the BAM was 100 fs peak-to-peak over $4 \mathrm{~h}$. By measuring the laser $\left(80 \mathrm{~nm}, 40 \mathrm{fs}\right.$ FWHM, $\left.1 \mathrm{~mJ} \mathrm{~cm}^{-2}\right)$ induced $\mathrm{Bi}(111)$ diffraction peak using the short bunch setting, the coherent $A_{1 g}$ phonon oscillation is clearly noticed as shown in Fig. 7. The X-ray beam was focused to $<10 \mu \mathrm{m}$ horizontally to minimize geometric time broadening. The instrumental time resolution is about $260 \mathrm{fs}$ FWHM without applying jitter or drift corrections for the X-ray time arrival, but applying drift correction using the LAM arrival information of the pump laser pulses. The remaining jitter is mainly due to the synchronization of the pump laser which was accurate to $190 \mathrm{fs}$ FWHM. In the meantime, the synchronization of the pump laser has been improved to $35 \mathrm{fs}$ FWHM. Laser-induced pump-probe experiments with time resolution $<200 \mathrm{fs}$ FWHM can therefore be performed without the need for shotto-shot timing corrections. This is due to the low timing jitter of the electron beam as also noticed at other XFEL facilities (Kang et al., 2017). Broadband single-cycle THz pulses at centre frequency near $1 \mathrm{THz}$ were generated through optical rectification of NIR pulses $(2.4 \mathrm{~mJ})$ at $1.3 \mu \mathrm{m}$ in an $\mathrm{OH} 1$ organic crystal. The electrical field strength measured by electro-optic sampling was $350 \mathrm{kV} \mathrm{cm}^{-1}$. A two-step procedure was followed to ensure overlap of the X-ray and $\mathrm{THz}$ pulses on the sample (Grübel et al., 2016). By using a collinear pump-probe geometry on a (010)-oriented $\mathrm{Sn}_{2} \mathrm{P}_{2} \mathrm{~S}_{6}$ bulk crystal the structural dynamics of the soft phonon mode has been probed by the $(332)$ and $(\overline{3} 3 \overline{2})$ diffraction peaks via coplanar asymmetric diffraction. The incidence angle was $15^{\circ}$ to limit the X-ray penetration depth. The measured response shown in Fig. 8 is in agreement with earlier measurements showing that THz-pumped tr-(R)XRD experiments can now be performed at Bernina.

\section{Conclusions}

The SwissFEL Aramis Bernina instrument is designed as a versatile tool for femtosecond pump-probe X-ray experiments in condensed matter physics and material science to 


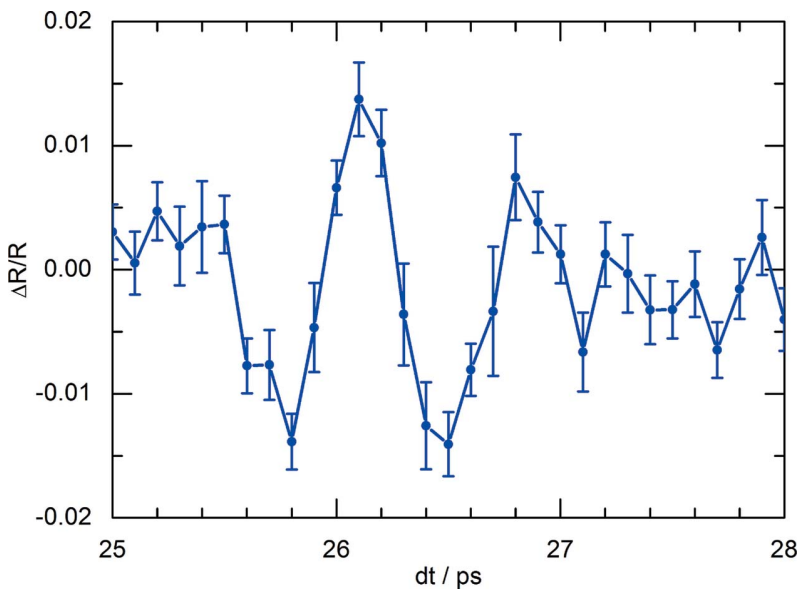

Figure 8

Time evolution of the normalized diffraction intensity of the (332) reflection in the ferroelectric $\mathrm{Sn}_{2} \mathrm{P}_{2} \mathrm{~S}_{6}$ induced by a $\mathrm{THz}$ electric field of $350 \mathrm{kV} \mathrm{cm}^{-1}$. Data were acquired during the second Bernina pilot experiment.

explore the coupling dynamics between atomic, electronic and magnetic constituents that lead to novel functional properties in materials. Emphasis is on developing selective excitation and probe techniques to stimulate and observe the response of the system when at the same time it is adiabatically tuned under extreme conditions. First pilot experiments have been performed to demonstrate IR and THz-pumped tr-XRD experiments with time resolution of about $260 \mathrm{fs}$ FWHM, limited by the arrival-time jitter between the X-ray and laser beams. Instrumentation to accurately measure pulse-by-pulse the X-ray intensity and spectrum, as well as the relative X-ray arrival, is under commissioning in order to exploit the timeresolved potential of Aramis.

\section{Acknowledgements}

Many colleaques at PSI have contributed to the technical design and construction of the Bernina experimental station. In alphabetical order: M. Brücker, R. Gaiffi, E. Huesler, S. Hunziker, M. Kaiser, A. Keller, C. Milne, C. Pradervand, B. Schmitt, P. Wiegand. The authors would like to extend their profound thanks to all of the facilities for collaboration, advice and support on the Bernina and entire SwissFEL project: DESY Hamburg (Germany), European XFEL (Schenefeld, Germany), ESRF (Grenoble, France) and SPring-8 (JASRI/ RIKEN, Hyogo, Japan). The authors would like to acknowledge the funding and support received from the Swiss Federal Government, the ETH Council, the government of the Canton of Aargau, and the Swiss National Science Foundation. The Bernina staff would like to further thank Klaus Sokolowski Tinten and Michael Horn-von Hoegen for providing thin film Bi samples for timing characterization. Finally we would like to acknowledge the support and effort from the numerous persons and entire groups at PSI who have contributed to this project.

\section{Funding information}

Funding for this research was provided by: Swiss National Government; ETH Council; Swiss National Science Foundation.

\section{References}

Amann, J., Berg, W., Blank, V., Decker, F.-J., Ding, Y., Emma, P., Feng, Y., Frisch, J., Fritz, D., Hastings, J., Huang, Z., Krzywinski, J., Lindberg, R., Loos, H., Lutman, A., Nuhn, H.-D., Ratner, D., Rzepiela, J., Shu, D., Shvyd'ko, Y., Spampinati, S., Stoupin, S., Terentyev, S., Trakhtenberg, E., Walz, D., Welch, J., Wu, J., Zholents, A. \& Zhu, D. (2012). Nat. Photon. 6, 693-698.

Angelovski, A., Kuntzsch, M., Czwalinna, M. K., Penirschke, A., Hansli, M., Sydlo, C., Arsov, V., Hunziker, S., Schlarb, H., Gensch, M., Schlott, V., Weiland, T. \& Jakoby, R. (2015). Phys. Rev. ST Accel. Beams, 18, 012801.

Arsov, V., Aiba, M., Dehler, M., Frei, F., Hunziker, S., Kaiser, M., Romann, A. \& Schlott, V. (2014). Proceedings of the 36th International Free Electron Laser Conference (FEL2014), 25-29 August 2014, Basel, Switzerland, pp. 933-936. THP085.

Beaud, P., Caviezel, A., Mariager, S. O., Rettig, L., Ingold, G., Dornes, C., Huang, S.-W., Johnson, J. A., Radovic, M., Huber, T., Kubacka, T., Ferrer, A., Lemke, H. T., Chollet, M., Zhu, D., Glownia, J. M., Sikorski, M., Robert, A., Wadati, H., Nakamura, M., Kawasaki, M., Tokura, Y., Johnson, S. L. \& Staub, U. (2014). Nat. Mater. 13, $923-$ 927.

Beaud, P., Johnson, S. L., Streun, A., Abela, R., Abramsohn, D., Grolimund, D., Krasniqi, F., Schmidt, T., Schlott, V. \& Ingold, G. (2007). Phys. Rev. Lett. 99, 174801.

Beaud, P., Johnson, S. L., Vorobeva, E., Staub, U., De Souza, R. A., Milne, C. J., Jia, Q. X. \& Ingold, G. (2009). Phys. Rev. Lett. 103, 155702.

Bionta, M. R., Lemke, H. T., Cryan, J. P., Glownia, J. M., Bostedt, C., Cammarata, M., Castagna, J.-C., Ding, Y., Fritz, D. M., Fry, A. R., Krzywinski, J., Messerschmidt, M., Schorb, S., Swiggers, M. L. \& Coffee, R. N. (2011). Opt. Express, 19, 21855-21865.

Bohnenbuck, B., Zegkinoglou, I., Strempfer, J., Schüßler-Langeheine, C., Nelson, C. S., Leininger, Ph., Wu, H.-H., Schierle, E., Lang, J. C., Srajer, G., Ikeda, S. I., Yoshida, Y., Iwata, K., Katano, S., Kikugawa, N. \& Keimer, B. (2008). Phys. Rev. B, 77, 224412.

Calvi, M., Camenzuli, C., Ganter, R., Sammut, N. \& Schmidt, Th. (2018). J. Synchrotron Rad. 25, 686-705.

Cartella, A., Nova, T. F., Oriana, A., Cerullo, G., Först, M., Manzoni, C. \& Cavalleri, A. (2017). Opt. Lett. 42, 663-666.

Chang, J., Blackburn, E., Holmes, A. T., Christensen, N. B., Larsen, J., Mesot, J., Liang, R., Bonn, D. A., Hardy, W. N., Watenphul, A., Zimmermann, M., Forgan, E. M. \& Hayden, S. M. (2012). Nat. Phys. 8, 871-876.

Chang, J., Blackburn, E., Ivashko, O., Holmes, A. T., Christensen, N. B., Hücker, M., Liang, R., Bonn, D. A., Hardy, W. N., Rütt, U., Zimmermann, M., Forgan, E. M. \& Hayden, S. M. (2016). Nat. Commun. 7, 11494.

Chen, F., Zhu, Y., Liu, S., Qi, Y., Hwang, H. Y., Brandt, N. C., Lu, J., Quirin, F., Enquist, H., Zalden, P., Hu, T., Goodfellow, J., Sher, M.-J., Hoffmann, M. C., Zhu, D., Lemke, H., Glownia, J., Chollet, M., Damodaran, A. R., Park, J., Cai, Z., Jung, I. W., Highland, M. J., Walko, D. A., Freeland, J. W., Evans, P. G., Vailionis, A., Larsson, J., Nelson, K. A., Rappe, A. M., Sokolowski-Tinten, K., Martin, L. W., Wen, H. \& Lindenberg, A. M. (2016). Phys. Rev. B, 94, 180104.

Chollet, M., Alonso-Mori, R., Cammarata, M., Damiani, D., Defever, J., Delor, J. T., Feng, Y., Glownia, J. M., Langton, J. B., Nelson, S., Ramsey, K., Robert, A., Sikorski, M., Song, S., Stefanescu, D., Srinivasan, V., Zhu, D., Lemke, H. T. \& Fritz, D. M. (2015). J. Synchrotron Rad. 22, 503-507.

Clark, J. N., Beitra, L., Xiong, G., Higginbotham, A., Fritz, D. M., Lemke, H. T., Zhu, D., Chollet, M., Williams, G. J., Messerschmidt, 
M., Abbey, B., Harder, R. J., Korsunsky, A. M., Wark, J. S. \& Robinson, I. K. (2013). Science, 341, 56-59.

Csatari Divall, M., Mutter, P., Divall, E. J. \& Hauri, C. P. (2015). Opt. Express, 23, 29929-29939.

Dean, M. P. M., Cao, Y., Liu, X., Wall, S., Zhu, D., Mankowsky, R., Thampy, V., Chen, X. M., Vale, J. G., Casa, D., Kim, J., Said, A. H., Juhas, P., Alonso-Mori, R., Glownia, J. M., Robert, A., Robinson, J., Sikorski, M., Song, S., Kozina, M., Lemke, H., Patthey, L., Owada, S., Katayama, T., Yabashi, M., Tanaka, Y., Togashi, T., Liu, J., Rayan Serrao, C., Kim, B. J., Huber, L., Chang, C.-L., McMorrow, D. F., Först, M. \& Hill, J. P. (2016). Nat. Mater. 15, 1-6.

Donnerer, C., Rahn, M. C., Sala, M. M., Vale, J. G., Pincini, D., Strempfer, J., Krisch, M., Prabhakaran, D., Boothroyd, A. T. \& McMorrow, D. F. (2016). Phys. Rev. Lett. 117, 037201.

Emma, P., Akre, R., Arthur, J., Bionta, R., Bostedt, C., Bozek, J., Brachmann, A., Bucksbaum, P., Coffee, R., Decker, F.-J., Ding, Y., Dowell, D., Edstrom, S., Fisher, A., Frisch, J., Gilevich, S., Hastings, J., Hays, G., Hering, P., Huang, Z., Iverson, R., Loos, H., Messerschmidt, M., Miahnahri, A., Moeller, S., Nuhn, H.-D., Pile, G., Ratner, D., Rzepiela, J., Schultz, D., Smith, T., Stefan, P., Tompkins, H., Turner, J., Welch, J., White, W., Wu, J., Yocky, G. \& Galayda, J. (2010). Nat. Photon. 4, 641-647.

Erny, C. \& Hauri, C. P. (2016). J. Synchrotron Rad. 23, 1143-1150.

Esposito, V., Fechner, M., Mankowsky, R., Lemke, H., Chollet, M., Glownia, J. M., Nakamura, M., Kawasaki, M., Tokura, Y., Staub, U., Beaud, P. \& Först, M. (2017). Phys. Rev. Lett. 118, 247601.

Fausti, D., Tobey, R. I., Dean, N., Kaiser, S., Dienst, A., Hoffmann, M. C., Pyon, S., Takayama, T., Takagi, H. \& Cavalleri, A. (2011). Science, 331, 189-191.

Feld, G. K., Heymann, M., Benner, W. H., Pardini, T., Tsai, C.-J., Boutet, S., Coleman, M. A., Hunter, M. S., Li, X., Messerschmidt, M., Opathalage, A., Pedrini, B., Williams, G. J., Krantz, B. A., Fraden, S., Hau-Riege, S., Evans, J. E., Segelke, B. W. \& Frank, M. (2015). J. Appl. Cryst. 48, 1072-1079.

Feng, Y., Wang, J., Jaramillo, R., van Wezel, J., Haravifard, S., Srajer, G., Liu, Y., Xu, Z.-A., Littlewood, P. B. \& Rosenbaum, T. F. (2012). Proc. Natl Acad. Sci. USA, 109, 7224-7229.

Follath, R., Flechsig, U., Milne, C., Szlachetko, J., Ingold, G., Patterson, B., Patthey, L. \& Abela, R. (2016). AIP Conf. Proc. 1741, 020009 .

Först, M., Caviglia, A. D., Scherwitzl, R., Mankowsky, R., Zubko, P., Khanna, V., Bromberger, H., Wilkins, S. B., Chuang, Y.-D., Lee, W. S., Schlotter, W. F., Turner, J. J., Dakovski, G. L., Minitti, M. P., Robinson, J., Clark, S. R., Jaksch, D., Triscone, J.-M., Hill, J. P., Dhesi, S. S. \& Cavalleri, A. (2015). Nat. Mater. 14, 883-888.

Först, M., Manzoni, C., Kaiser, S., Tomioka, Y., Tokura, Y., Merlin, R. \& Cavalleri, A. (2011a). Nat. Phys. 7, 854-856.

Först, M., Tobey, R. I., Wall, S., Bromberger, H., Khanna, V., Cavalieri, A. L., Chuang, Y.-D., Lee, W. S., Moore, R., Schlotter, W. F., Turner, J. J., Krupin, O., Trigo, M., Zheng, H., Mitchell, J. F., Dhesi, S. S., Hill, J. P. \& Cavalleri, A. (2011b). Phys. Rev. B, 84, 241104.

Francoual, S., Strempfer, J., Reuther, D., Shukla, D. K. \& Skaugen, A. (2013). J. Phys. Conf. Ser. 425, 132010.

Gerber, S., Jang, H., Nojiri, H., Matsuzawa, S., Yasumura, H., Bonn, D. A., Liang, R., Hardy, W. N., Islam, Z., Mehta, A., Song, S., Sikorski, M., Stefanescu, D., Feng, Y., Kivelson, S. A., Devereaux, T. P., Shen, Z.-X., Kao, C.-C., Lee, W.-S., Zhu, D. \& Lee, J.-S. (2015). Science, 350, 949-952.

Gibbs, D., Harshman, D. R., Isaacs, E. D., McWhan, D. B., Mills, D. \& Vettier, C. (1988). Phys. Rev. Lett. 61, 1241-1244.

Gog, T., Casa, D. M., Said, A. H., Upton, M. H., Kim, J., Kuzmenko, I., Huang, X. \& Khachatryan, R. (2013). J. Synchrotron Rad. 20, 7479.

Gorgisyan, I., Ischebeck, R., Erny, C., Dax, A., Patthey, L., Pradervand, C., Sala, L., Milne, C., Lemke, H. T., Hauri, C. P., Katayama, T., Owada, S., Yabashi, M., Togashi, T., Abela, R., Rivkin, L. \& Juranić, P. (2017). Opt. Express, 25, 2080-2091.
Gray, A. X., Hoffmann, M. C., Jeong, J., Aetukuri, N. P., Zhu, D., Hwang, H. Y., Brandt, N. C., Wen, H., Sternbach, A. J., Bonetti, S., Reid, A. H., Kukreja, R., Graves, C., Wang, T., Granitzka, P., Chen, Z., Higley, D. J., Chase, T., Jal, E., Abreu, E., Liu, M. K., Weng, T.-C., Sokaras, D., Nordlund, D., Chollet, M., Alonso-Mori, R., Lemke, H., Glownia, J. M., Trigo, M., Zhu, Y., Ohldag, H., Freeland, J. W., Samant, M. G., Berakdar, J., Averitt, R. D., Nelson, K. A., Parkin, S. S. P. \& Dürr, H. A. (2018). Phys. Rev. B, 98, 045104

Grenier, S., Hill, J. P., Gibbs, D., Thomas, K. J., Zimmermann, M., Nelson, C. S., Kiryukhin, V., Tokura, Y., Tomioka, Y., Casa, D., Gog, T. \& Venkataraman, C. (2004). Phys. Rev. B, 69, 134419.

Grübel, S., Johnson, J. A., Beaud, P., Dornes, C., Ferrer, A., Haborets, V., Huber, L., Huber, T., Kohutych, A., Kubacka, T., Kubli, M., Mariager, S. O., Rittmann, J., Saari, J. I., Vysochanskii, Y., Ingold, G. \& Johnson, S. L. (2016). arXiv:1602.05435.

Haravifard, S., Banerjee, A., Lang, J. C., Srajer, G., Silevitch, D. M., Gaulin, B. D., Dabkowska, H. A. \& Rosenbaum, T. F. (2012). Proc. Natl Acad. Sci. 109, 2286-2289.

Harmand, M., Coffee, R., Bionta, M. R., Chollet, M., French, D., Zhu, D., Fritz, D. M., Lemke, H. T., Medvedev, N., Ziaja, B., Toleikis, S. \& Cammarata, M. (2013). Nat. Photon. 7, 215-218.

Hartmann, N., Helml, W., Galler, A., Bionta, M. R., Grünert, J., Molodtsov, S., Ferguson, K. R., Schorb, S., Swiggers, M. L., Carron, S., Bostedt, C., Castagna, J.-C., Bozek, J., Glownia, J. M., Kane, D. J., Fry, A. R., White, W. E., Hauri, C. P., Feurer, T. \& Coffee, R. N. (2014). Nat. Photon. 8, 706-709.

Hauri, C. P., Ruchert, C., Vicario, C. \& Ardana, F. (2011). Appl. Phys. Lett. 99, 161116.

Hebling, J., Almasi, G., Kozma, I. \& Kuhl, J. (2002). Opt. Express, 10, 1161-1166.

Hernandez, A. S., Prat, E., Bettoni, S., Beutner, B. \& Reiche, S. (2016). Phys. Rev. Accel. Beams, 19, 090702.

Hirata, K., Shinzawa-Itoh, K., Yano, N., Takemura, S., Kato, K., Hatanaka, M., Muramoto, K., Kawahara, T., Tsukihara, T., Yamashita, E., Tono, K., Ueno, G., Hikima, T., Murakami, H., Inubushi, Y., Yabashi, M., Ishikawa, T., Yamamoto, M., Ogura, T., Sugimoto, H., Shen, J.-R., Yoshikawa, S. \& Ago, H. (2014). Nat. Methods, 11, 734-736.

Hoegen, A. von, Mankowsky, R., Fechner, M., Först, M. \& Cavalleri, A. (2018). Nature, 555, 79-82.

Hu, W., Kaiser, S., Nicoletti, D., Hunt, C. R., Gierz, I., Hoffmann, M. C., Le Tacon, M., Loew, T., Keimer, B. \& Cavalleri, A. (2014). Nat. Mater. 13, 705-711.

Ingold, G., Abela, R., Beaud, P., Johnson, S. L. \& Staub, U. (2008). Z. Kristallogr. 223, 292-306.

Ingold, G. \& Beaud, P. (2013). SwissFEL Experimental Station B. Concept Design Report. SwissFEL, Paul Scherrer Institute, Villigen, Switzerland.

Ingold, G., Rittmann, J., Beaud, P., Divall, M., Erny, C., Flechsig, U., Follath, R., Hauri, C. P., Hunziker, S., Juranic, P., Mozzanica, A., Pedrini, B., Sala, L., Patthey, L., Patterson, B. D. \& Abela, R. (2016). AIP Conf. Proc. 1741, 030039.

Ishikawa, T., Aoyagi, H., Asaka, T., Asano, Y., Azumi, N., Bizen, T., Ego, H., Fukami, K., Fukui, T., Furukawa, Y., Goto, S., Hanaki, H., Hara, T., Hasegawa, T., Hatsui, T., Higashiya, A., Hirono, T., Hosoda, N., Ishii, M., Inagaki, T., Inubushi, Y., Itoga, T., Joti, Y., Kago, M., Kameshima, T., Kimura, H., Kirihara, Y., Kiyomichi, A., Kobayashi, T., Kondo, C., Kudo, T., Maesaka, H., Maréchal, X. M., Masuda, T., Matsubara, S., Matsumoto, T., Matsushita, T., Matsui, S., Nagasono, M., Nariyama, N., Ohashi, H., Ohata, T., Ohshima, T., Ono, S., Otake, Y., Saji, C., Sakurai, T., Sato, T., Sawada, K., Seike, T., Shirasawa, K., Sugimoto, T., Suzuki, S., Takahashi, S., Takebe, H., Takeshita, K., Tamasaku, K., Tanaka, H., Tanaka, R., Tanaka, T., Togashi, T., Togawa, K., Tokuhisa, A., Tomizawa, H., Tono, K., Wu, S., Yabashi, M., Yamaga, M., Yamashita, A., Yanagida, K., Zhang, C., Shintake, T., Kitamura, H. \& Kumagai, N. (2012). Nat. Photon. 6, 540-544. 
Jang, H., Lee, W.-S., Nojiri, H., Matsuzawa, S., Yasumura, H., Nie, L., Maharaj, A. V., Gerber, S., Liu, Y.-J., Mehta, A., Bonn, D. A., Liang, R., Hardy, W. N., Burns, C. A., Islam, Z., Song, S., Hastings, J., Devereaux, T. P., Shen, Z.-X., Kivelson, S. A., Kao, C.-C., Zhu, D. \& Lee, J.-S. (2016). Proc. Natl Acad. Sci. 113, 14645-14650.

Jaramillo, R., Feng, Y., Lang, J. C., Islam, Z., Srajer, G., Littlewood, P. B., McWhan, D. B. \& Rosenbaum, T. F. (2009). Nature, 459, 405409.

Johnson, J. A., Kubacka, T., Hoffmann, M. C., Vicario, C., de Jong, S., Beaud, P., Grübel, S., Huang, S.-W., Huber, L., Windsor, Y. W., Bothschafter, E. M., Rettig, L., Ramakrishnan, M., Alberca, A., Patthey, L., Chuang, Y.-D., Turner, J. J., Dakovski, G. L., Lee, W.-S., Minitti, M. P., Schlotter, W., Moore, R. G., Hauri, C. P., Koohpayeh, S. M., Scagnoli, V., Ingold, G., Johnson, S. L. \& Staub, U. (2015). Phys. Rev. B, 92, 184429.

Johnson, S. L., Beaud, P., Milne, C. J., Krasniqi, F. S., Zijlstra, E. S., Garcia, M. E., Kaiser, M., Grolimund, D., Abela, R. \& Ingold, G. (2008). Phys. Rev. Lett. 100, 155501.

Johnson, S. L., Beaud, P., Vorobeva, E., Milne, C. J., Murray, É. D., Fahy, S. \& Ingold, G. (2010). Acta Cryst. A66, 157-167.

Johnson, S. L., de Souza, R. A., Staub, U., Beaud, P., Möhr-Vorobeva, E., Ingold, G., Caviezel, A., Scagnoli, V., Schlotter, W. F., Turner, J. J., Krupin, O., Lee, W.-S., Chuang, Y.-D., Patthey, L., Moore, R. G., Lu, D., Yi, M., Kirchmann, P. S., Trigo, M., Denes, P., Doering, D., Hussain, Z., Shen, Z.-X., Prabhakaran, D. \& Boothroyd, A. T. (2012). Phys. Rev. Lett. 108, 037203.

Kang, H.-S., Min, C.-K., Heo, H., Kim, C., Yang, H., Kim, G., Nam, I., Baek, S. Y., Choi, H.-J., Mun, G., Park, B. R., Suh, Y. J., Shin, D. C., Hu, J., Hong, J., Jung, S., Kim, S.-H., Kim, K., Na, D., Park, S. S., Park, Y. J., Han, J.-H., Jung, Y. G., Jeong, S. H., Lee, H. G., Lee, S., Lee, S., Lee, W.-W., Oh, B., Suh, H. S., Parc, Y. W., Park, S.-J., Kim, M. H., Jung, N.-S., Kim, Y.-C., Lee, M.-S., Lee, B.-H., Sung, C.-W., Mok, I.-S., Yang, J.-M., Lee, C.-S., Shin, H., Kim, J. H., Kim, Y., Lee, J. H., Park, S.-Y., Kim, J., Park, J., Eom, I., Rah, S., Kim, S., Nam, K. H., Park, J., Park, J., Kim, S., Kwon, S., Park, S. H., Kim, K. S., Hyun, H., Kim, S. N., Kim, S., Hwang, S., Kim, M. J., Lim, C., Yu, C.-J., Kim, B.-S., Kang, T.-H., Kim, K.-W., Kim, S.-H., Lee, H.-S., Lee, H.-S., Park, K.-H., Koo, T.-Y., Kim, D.-E. \& Ko, I. S. (2017). Nat. Photon. 11, 708-713.

Kern, J., Alonso-Mori, R., Tran, R., Hattne, J., Gildea, R. J., Echols, N., Glöckner, C., Hellmich, J., Laksmono, H., Sierra, R. G., Lassalle-Kaiser, B., Koroidov, S., Lampe, A., Han, G., Gul, S., Difiore, D., Milathianaki, D., Fry, A. R., Miahnahri, A., Schafer, D. W., Messerschmidt, M., Seibert, M. M., Koglin, J. E., Sokaras, D., Weng, T.-C., Sellberg, J., Latimer, M. J., Grosse-Kunstleve, R. W., Zwart, P. H., White, W. E., Glatzel, P., Adams, P. D., Bogan, M. J., Williams, G. J., Boutet, S., Messinger, J., Zouni, A., Sauter, N. K., Yachandra, V. K., Bergmann, U. \& Yano, J. (2013). Science, 340, 491-495.

Kim, J., Casa, D., Upton, M. H., Gog, T., Kim, Y.-J., Mitchell, J. F., van Veenendaal, M., Daghofer, M., van den Brink, J., Khaliullin, G. \& Kim, B. J. (2011). Phys. Rev. Lett. 108, 177003.

Kim, J. W., Choi, Y., Kim, J., Mitchell, J. F., Jackeli, G., Daghofer, M., van den Brink, J., Khaliullin, G. \& Kim, B. J. (2012). Phys. Rev. Lett. 109, 037204.

Kim, K. H., Späh, A., Pathak, H., Perakis, F., Mariedahl, D., AmannWinkel, K., Sellberg, J. A., Lee, J. H., Kim, S., Park, J., Nam, K. H., Katayama, T. \& Nilsson, A. (2017). Science, 358, 1589-1593.

Kozina, M., Pancaldi, M., Bernhard, C., van Driel, T., Glownia, J. M., Marsik, P., Radovic, M., Vaz, C. A. F., Zhu, D., Bonetti, S., Staub, U. \& Hoffmann, M. C. (2017b). Appl. Phys. Lett. 110, 081106.

Kozina, M., van Driel, T., Chollet, M., Sato, T., Glownia, J. M., Wandel, S., Radovic, M., Staub, U. \& Hoffmann, M. C. (2017a). Struct. Dyn. 4, 054301.

Kubacka, T., Johnson, J., Hoffmann, M. C., Vicario, C., de Jong, S., Beaud, P., Grübel, S., Huang, S.-W., Huber, L., Patthey, L., Chuang, Y.-D., Turner, J. J., Dakovski, G. L., Lee, W.-S., Minitti, M. P., Schlotter, W., Moore, R. G., Hauri, C. P., Koohpayeh, S. M.,
Scagnoli, V., Ingold, G., Johnson, S. L. \& Staub, U. (2014). Science, 343, 1333-1336.

Liu, B., Bromberger, H., Cartella, A., Gebert, T., Först, M. \& Cavalleri, A. (2017). Opt. Lett. 42, 129-131.

Lutman, A. A., MacArthur, J. P., Ilchen, M., Lindahl, A. O., Buck, J., Coffee, R. N., Dakovski, G. L., Dammann, L., Ding, Y., Dürr, H. A., Glaser, L., Grünert, J., Hartmann, G., Hartmann, N., Higley, D., Hirsch, K., Levashov, Y. I., Marinelli, A., Maxwell, T., Mitra, A., Moeller, S., Osipov, T., Peters, F., Planas, M., Shevchuk, I., Schlotter, W. F., Scholz, F., Seltmann, J., Viefhaus, J., Walter, P., Wolf, Z. R., Huang, Z. \& Nuhn, H.-D. (2016). Nat. Photon. 10, 468472.

Mankowsky, R., Subedi, A., Först, M., Mariager, S. O., Chollet, M., Lemke, H. T., Robinson, J. S., Glownia, J. M., Minitti, M. P., Frano, A., Fechner, M., Spaldin, N., Loew, T., Keimer, B., Georges, A. \& Cavalleri, A. (2014). Nature, 516, 71-73.

Mankowsky, R., von Hoegen, A., Först, M. \& Cavalleri, A. (2017). Phys. Rev. Lett. 118, 197601.

Mazzoli, C., Wilkins, S. B., Di Matteo, S., Detlefs, B., Detlefs, C., Scagnoli, V., Paolasini, L. \& Ghigna, P. (2007). Phys. Rev. B, 76, 195118.

Milne, C., Schietinger, T., Aiba, M., Alarcon, A., Alex, J., Anghel, A., Arsov, V., Beard, C., Beaud, P., Bettoni, S., Bopp, M., Brands, H., Brönnimann, M., Brunnenkant, I., Calvi, M., Citterio, A., Craievich, P., Csatari Divall, M., Dällenbach, M., D’Amico, M., Dax, A., Deng, Y., Dietrich, A., Dinapoli, R., Divall, E., Dordevic, S., Ebner, S., Erny, C., Fitze, H., Flechsig, U., Follath, R., Frei, F., Gärtner, F., Ganter, R., Garvey, T., Geng, Z., Gorgisyan, I., Gough, C., Hauff, A., Hauri, C., Hiller, N., Humar, T., Hunziker, S., Ingold, G., Ischebeck, R., Janousch, M., Juranić, P., Jurcevic, M., Kaiser, M., Kalantari, B., Kalt, R., Keil, B., Kittel, C., Knopp, G., Koprek, W., Lemke, H., Lippuner, T., Llorente Sancho, D., Löhl, F., LopezCuenca, C., Märki, F., Marcellini, F., Marinkovic, G., Martiel, I., Menzel, R., Mozzanica, A., Nass, K., Orlandi, G., Ozkan Loch, C., Panepucci, E., Paraliev, M., Patterson, B., Pedrini, B., Pedrozzi, M., Pollet, P., Pradervand, C., Prat, E., Radi, P., Raguin, J., Redford, S., Rehanek, J., Réhault, J., Reiche, S., Ringele, M., Rittmann, J., Rivkin, L., Romann, A., Ruat, M., Ruder, C., Sala, L., Schebacher, L., Schilcher, T., Schlott, V., Schmidt, T., Schmitt, B., Shi, X., Stadler, M., Stingelin, L., Sturzenegger, W., Szlachetko, J., Thattil, D., Treyer, D., Trisorio, A., Tron, W., Vetter, S., Vicario, C., Voulot, D., Wang, M., Zamofing, T., Zellweger, C., Zennaro, R., Zimoch, E., Abela, R., Patthey, L. \& Braun, H. (2017). Appl. Sci. 7, 720.

Mitrano, M., Cantaluppi, A., Nicoletti, D., Kaiser, S., Perucchi, A., Lupi, S., Di Pietro, P., Pontiroli, D., Riccò, M., Clark, S. R., Jaksch, D. \& Cavalleri, A. (2016). Nature, 530, 461-464.

Moretti Sala, M., Martel, K., Henriquet, C., Al Zein, A., Simonelli, L., Sahle, C., Gonzalez, H., Lagier, M.-C., Ponchut, C., Huotari, S., Verbeni, R., Krisch, M. \& Monaco, G. (2018). J. Synchrotron Rad. 25, 580-591.

Mozzanica, A., Andrä, M., Barten, R., Bergamaschi, A., Chiriotti, S., Brückner, M., Dinapoli, R., Fröjdh, E., Greiffenberg, D., Leonarski, F., Lopez-Cuenca, C., Mezza, D., Redford, S., Ruder, C., Schmitt, B., Shi, X., Thattil, D., Tinti, G., Vetter, S. \& Zhang, J. (2018). Synchrotron Radiat. News, 31(6), 16-20.

Mozzanica, A., Bergamaschi, A., Cartier, S., Dinapoli, R., Greiffenberg, D., Johnson, I., Jungmann, J., Maliakal, D., Mezza, D., Ruder, C., Schaedler, L., Schmitt, B., Shi, X. \& Tinti, G. (2014). J. Instrum. 9, C05010.

Murakami, Y., Hill, J., Gibbs, D., Blume, M., Koyama, I., Tanaka, M., Kawata, H., Arima, T., Tokura, Y., Hirota, K. \& Endoh, Y. (1998a). Phys. Rev. Lett. 81, 582-585.

Murakami, Y., Kawada, H., Kawata, H., Tanaka, M., Arima, T., Moritomo, Y. \& Tokura, Y. (1998b). Phys. Rev. Lett. 80, 1932-1935. Nisoli, M., De Silvestri, S. \& Svelto, O. (1996). Appl. Phys. Lett. 68, 2793-2795.

Opara, N., Martiel, I., Arnold, S. A., Braun, T., Stahlberg, H., Makita, M., David, C. \& Padeste, C. (2017). J. Appl. Cryst. 50, 909-918. 
Paolasini, L., Detlefs, C., Mazzoli, C., Wilkins, S., Deen, P. P., Bombardi, A., Kernavanois, N., de Bergevin, F., Yakhou, F., Valade, J. P., Breslavetz, I., Fondacaro, A., Pepellin, G. \& Bernard, P. (2007). J. Synchrotron Rad. 14, 301-312.

Pedrini, B., Martiel, I., Panepucci, E. \& Pradervand, C. (2017). SwissFEL ESB-MX instrument for fixed target protein crystallography at SwissFEL. Concept Design Report. SwissFEL, Paul Scherrer Institute, Villigen, Switzerland.

Prat, E. \& Reiche, S. (2013). Proceedings of the 35th Free-Electron Laser Conference (FEL2013), 26-30 August 2013, New York, NY, USA, pp. 618-622.

Rehanek, J., Makita, M., Wiegand, P., Heimgartner, P., Pradervand, C., Seniutinas, G., Flechsig, U., Thominet, V., Schneider, C., Fernandez, A. R., David, C., Patthey, L. \& Juranić, P. (2017). J. Instrum. 12, P05024.

Rettig, L., Dornes, C., Thielemann-Kühn, N., Pontius, N., Zabel, H., Schlagel, D. L., Lograsso, T. A., Chollet, M., Robert, A., Sikorski, M., Song, S., Glownia, J. M., Schüßler-Langeheine, C., Johnson, S. L. \& Staub, U. (2016). Phys. Rev. Lett. 116, 257202.

Roedig, P., Duman, R., Sanchez-Weatherby, J., Vartiainen, I., Burkhardt, A., Warmer, M., David, C., Wagner, A. \& Meents, A. (2016). J. Appl. Cryst. 49, 968-975.

Roedig, P., Vartiainen, I., Duman, R., Panneerselvam, S., Stübe, N., Lorbeer, O., Warmer, M., Sutton, G., Stuart, D. I., Weckert, E., David, C., Wagner, A. \& Meents, A. (2015). Sci. Rep. 5, 10451.

Ruchert, C., Vicario, C. \& Hauri, C. P. (2013). Phys. Rev. Lett. 110, 123902.

Scagnoli, V., Mazzoli, C., Detlefs, C., Bernard, P., Fondacaro, A., Paolasini, L., Fabrizi, F. \& de Bergevin, F. (2009). J. Synchrotron Rad. 16, 778-787.

Schietinger, T., Pedrozzi, M., Aiba, M., Arsov, V., Bettoni, S., Beutner, B., Calvi, M., Craievich, P., Dehler, M., Frei, F., Ganter, R., Hauri, C. P., Ischebeck, R., Ivanisenko, Y., Janousch, M., Kaiser, M., Keil, B., Löhl, F., Orlandi, G. L., Ozkan Loch, C., Peier, P., Prat, E., Raguin, J.-Y., Reiche, S., Schilcher, T., Wiegand, P., Zimoch, E., Anicic, D., Armstrong, D., Baldinger, M., Baldinger, R., Bertrand, A., Bitterli, K., Bopp, M., Brands, H., Braun, H. H., Brönnimann, M., Brunnenkant, I., Chevtsov, P., Chrin, J., Citterio, A., Csatari Divall, M., Dach, M., Dax, A., Ditter, R., Divall, E., Falone, A., Fitze, H., Geiselhart, C., Guetg, M. W., Hämmerli, F., Hauff, A., Heiniger, M., Higgs, C., Hugentobler, W., Hunziker, S., Janser, G.,
Kalantari, B., Kalt, R., Kim, Y., Koprek, W., Korhonen, T., Krempaska, R., Laznovsky, M., Lehner, S., Le Pimpec, F., Lippuner, T., Lutz, H., Mair, S., Marcellini, F., Marinkovic, G., Menzel, R., Milas, N., Pal, T., Pollet, P., Portmann, W., Rezaeizadeh, A., Ritt, S., Rohrer, M., Schär, M., Schebacher, L., Scherrer, S., Schlott, V., Schmidt, T., Schulz, L., Smit, B., Stadler, M., Steffen, B., Stingelin, L., Sturzenegger, W., Treyer, D. M., Trisorio, A., Tron, W., Vicario, C., Zennaro, R. \& Zimoch, D. (2016). Phys. Rev. Accel. Beams, 19, 100702.

Schmidt, T. \& Calvi, M. (2018). Synchrotron Radiat. News, 31(3), $35-40$.

Singer, A., Patel, S. K. K., Kukreja, R., Uhlír, V., Wingert, J., Festersen, S., Zhu, D., Glownia, J. M., Lemke, H. T., Nelson, S., Kozina, M., Rossnagel, K., Bauer, M., Murphy, B. M., Magnussen, O. M., Fullerton, E. E. \& Shpyrko, O. G. (2016). Phys. Rev. Lett. 117, 056401.

Strempfer, J., Francoual, S., Reuther, D., Shukla, D. K., Skaugen, A., Schulte-Schrepping, H., Kracht, T. \& Franz, H. (2013). J. Synchrotron Rad. 20, 541-549.

Suzuki, M., Inubushi, Y., Yabashi, M. \& Ishikawa, T. (2014). J. Synchrotron Rad. 21, 466-472.

Takahashi, H., Sugimoto, A., Nambu, Y., Yamauchi, T., Hirata, Y., Kawakami, T., Avdeev, M., Matsubayashi, K., Du, F., Kawashima, C., Soeda, H., Nakano, S., Uwatoko, Y., Ueda, Y., Sato, T. J. \& Ohgushi, K. (2015). Nat. Mater. 14, 1008-1012.

Tono, K., Kudo, T., Yabashi, M., Tachibana, T., Feng, Y., Fritz, D., Hastings, J. \& Ishikawa, T. (2011). Rev. Sci. Instrum. 82, 023108.

Trigo, M., Fuchs, M., Chen, J., Jiang, M. P., Cammarata, M., Fahy, S., Fritz, D. M., Gaffney, K., Ghimire, S., Higginbotham, A., Johnson, S. L., Kozina, M. E., Larsson, J., Lemke, H., Lindenberg, A. M., Ndabashimiye, G., Quirin, F., Sokolowski-Tinten, K., Uher, C., Wang, G., Wark, J. S., Zhu, D. \& Reis, D. A. (2013). Nat. Phys. 9, 790-794.

Yumoto, H., Mimura, H., Koyama, T., Matsuyama, S., Tono, K., Togashi, T., Inubushi, Y., Sato, T., Tanaka, T., Kimura, T., Yokoyama, H., Kim, J., Sano, Y., Hachisu, Y., Yabashi, M., Ohashi, H., Ohmori, H., Ishikawa, T. \& Yamauchi, K. (2013). Nat. Photon. 7, 43-47.

Zimmermann, M., Nelson, C. S., Hill, J. P., Gibbs, D., Blume, M., Casa, D., Keimer, B., Murakami, Y., Kao, C., Venkataraman, C., Gog, T., Tomioka, Y. \& Tokura, Y. (2001). Phys. Rev. B, 64, 195133. 